\title{
Generalized Anxiety Disorder Across the Lifespan: An Integrative Approach.
}

\section{Michael E. Portman: Publisher: New York: Springer, 2009}

Two aspects of this book are striking. The first is that "integrative approach" is not just a part of its title. Indeed, integration is the underlying philosophy of this work, which is, therefore, a truly integrative effort to present our current understanding of generalized anxiety disorder (GAD) and its treatment. Although a larger part of the book describes psychological models and psychological treatment of $\mathrm{GAD}$, there is adequate information on neurobiological approaches and pharmacological treatment, with a good balance achieved between the two. The second aspect of the book that I found striking and that I liked is that it often emphasizes what every good clinician knows, but is sorely missing from much of the contemporary literature in psychiatry and clinical psychology: the fact that our patients have different preferences and goals, a need for clinicians to respect that, be flexible, and adapt their treatment strategy to these preferences and goals, and the importance of the patient-therapist relationship and clinical experience.

Thus, this volume by Dr Portman, a clinical social worker and psychotherapist based in Cleveland, brings a refreshing perspective on one of the most intriguing and relatively neglected mental disorders. Dr Portman is to be congratulated for tackling $\mathrm{GAD}$, a condition that is an embarrassment to the mental health professionals, considering how prevalent it is, yet how often it is unrecognised or misdiagnosed and how poorly it is understood. Also, this book rightly emphasizes that there is no room for complacency when it comes to outcome of treatment and prognosis of this disorder, often erroneously presumed to be relatively "minor"

The structure of the book is logical. The first chapter presents clinical features and epidemiological data, while the second is concerned with the diagnosis of GAD and its assessment. Conceptual models of GAD are presented in chapter 3, which is followed by a presentation of the psychological treatments, most of which are based on these conceptual models. Chapter 5 is devoted to the genetics, neurobiology, and pharmacological treatment of GAD. Chapter 6 is one of the unique features of this book in that it discusses all aspects of GAD in children, adolescents, and the elderly - something that is often omitted from books on GAD or anxiety disorders in general. Chapter 7 focuses on "enigmas and paradoxes", of which there are many in GAD, but only a few have been selected for inclusion here. Quite appropriately, the last chapter looks at the future of GAD.

While the book provides much information that is factual and objective (e.g., regarding epidemiology and diagnosis), it also reflects the author's own views and opinions. The latter is a major strength of the book, because these views and opinions are well founded and also because they give this work a very personal touch, which makes reading it a pleasure. This is so delightfully different from books and other works that are faithful to the evidence-based dogma to the extent that they lack their own identity and come across as "dry", if not boring. Dr Portman's critical and wise voice is heard particularly well in the chapters on psychological treatment and pharmacotherapy of GAD. It is reassuring here that he refuses to be drawn in ideological battles fought by proponents of different schools of thought and that he acknowledges clearly and coherently the contributions and limitations of each therapeutic approach to GAD. This can be done only by someone who is both open-minded and has enough confidence based on sound clinical reasoning and experience.

Is something missing from this volume? Should the author have done something differently? There is no perfect book and this one is no exception. But how important are its "flaws"? In my view, problems with this book are minor and they are less in its content and some "structural decisions" than in the way the book presents its material. Surely, the content could have been enriched, for example, with greater attention to the nuances of the clinical presentation of $G A D$ and its multiple relationships with other forms of psychopathology. Perhaps more space should have been devoted to the links between GAD and depression, in view of the ongoing debates about the nature, significance, and implications of these links. The discussion of treatment-resistant GAD could have been included in the chapters on psychological treatment and pharmacotherapy rather than being a part of the "enigmas and paradoxes" chapter. Much information could have been presented in tables and figures, especially in chapters on the conceptual models, psychosocial treatments, and pharmacotherapy. This would have made it even easier to navigate through the book and it would have made the book more visually appealing. Also, a review of the studies of pharmacological and psychological treatments might have been more effective - pointing to practical implications - had it been more succinct, without describing the potentially distracting details of these studies.

Finally, each book should be judged in accordance with the degree to which its stated goals or intentions have been met. As already noted, the goal of achieving integration and balance has been met to the extent hardly seen elsewhere. The same could be said about the goal of producing a "resource for the busy clinician" and a "user-friendly guide". Indeed, this book is easy to read, it engages the reader and provides up-to-date information on most clinically relevant aspects of $\mathrm{GAD}$, and it is "down-to-earth" pragmatic. Small wonder, then, that in my assessment, Dr Portman's book is likely to be frequently consulted by clinicians assessing and treating people with GAD.

Vladan Starcevic Associate Professor, University of Sydney, Consultant Psychiatrist, Nepean Hospital, Sydney, Australia email: starcev@wahs.nsw.gov.au 